\title{
ABSORPTION OF SOLAR RADIATION AT THE ANTARCTIC SNOW SURFACE (Abstract)
}

\author{
by
}

Thomas C. Grenfell, Stephen G. Warren and Peter C. Mullen

(Department of Atmospheric Sciences AK-40, University of Washington, Seattle, WA 98195, U.S.A.)

\section{ABSTRACT}

Solar radiation incident on, and reflected by, the snow surface was measured near the South Pole as a function of wavelength, angle, and distance from the station. The objectives of the study were: (1) to observe spectral albedos of snow across the solar spectrum, (2) to obtain depth profiles of snow-grain radius in order to construct theoretical models of spectral albedo for pure snow, (3) to document the extent and degree of soot pollution due to station activities and to assess whether it could invalidate solar-radiation measurements made close to large stations, and (4) to obtain the spectral distribution of incident solar radiation at the Antarctic surface for various cloud conditions, in order to test radiation models of the Antarctic atmosphere.

Spectral albedo, measured under diffuse lighting

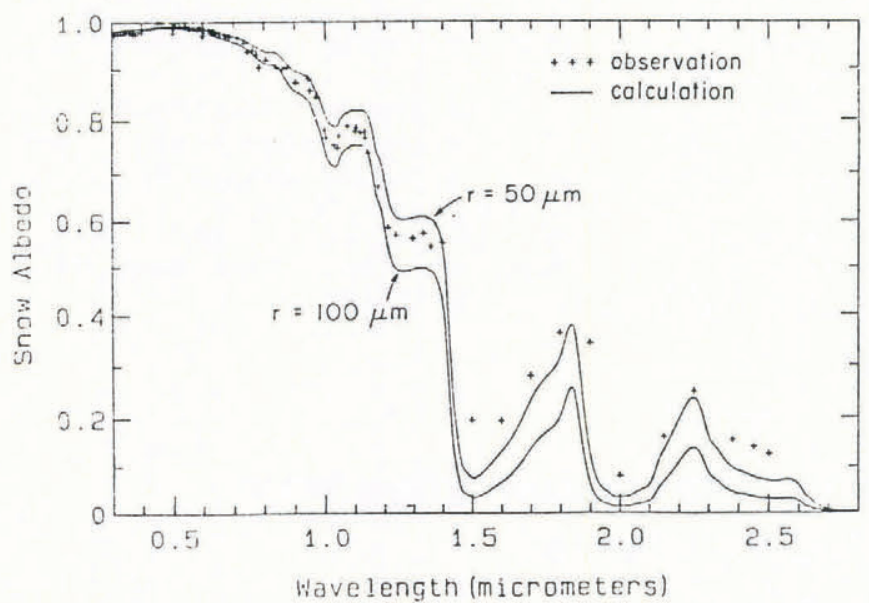

conditions (overcast cloud) on many days, repeatedly agreed with the results of theoretical models which predicted values approaching unity in the visible and found grain-size to be the most important variable controlling snow albedo in the near-infra-red. A representative albedo curve is shown in Figure 1. The visible albedo values were found to be $98-99 \%$ and were relatively insensitive to grain-size. (These results disagree with the only previous measurements of Antarctic snow albedo which had good spectral resolution: those of Kuhn and Siogas. Their maximum albedo was only about $90 \%$ in the visible.) The near-infra-red albedo, however, varied substantially among the experiments, due to day-to-day variations in snow grain-size, caused by precipitation and wind drifting. The experimental points in the figure match theoretical calculations for grain radius less than $50 \mu \mathrm{m}$ at wavelengths beyond $1.5 \mu \mathrm{m}$, and $50-100 \mu \mathrm{m}$ for shorter wavelengths. At the shorter wavelengths the light penetrates more deeply into the snow, so the albedo is sensitive to grains beneath the surface, whereas at the longer wavelengths the albedo is influenced only by the grains very close to the surface. The observed albedos can thus be explained by an increase in grain-size with depth.

In order that our measurements would be representative of large areas, we were concerned to avoid possible effects of pollution from the station. We collected samples from the top $20 \mathrm{~cm}$ of snow, melted and filtered them, and analyzed the filters. The conclusion is that the pollution is very minor. Just $500 \mathrm{~m}$ up-wind of the station there is normally less than $1 \mathrm{ng}$ of carbon per gram of snow $(1 \mathrm{ppb})$. Even down-wind of the station the carbon content did not exceed $3 \mathrm{ppb}$. For snow grain-sizes typical of Antarctica, our models predict that $15 \mathrm{ppb}$ carbon would reduce snow albedo by only $1 \%$ at the most sensitive wavelength. Thus we reject our earlier suggestion that the low visible albedos of Kuhn and Siogas were due to impurities in the snow and now favor other explanations. 